\title{
The right ventricle in patients with chronic heart failure and atrial fibrillation
}

\author{
Ewa Majos, Rafał Dąbrowski, Hanna Szwed \\ $2^{\text {nd }}$ Ischemic Heart Disease Department, Institute of Cardiology, Warsaw, Poland
}

\begin{abstract}
Under normal conditions function of the right ventricle $(R V)$ is determined by the heart rhythm, $R V$ filling time, $R V$ systolic synchrony and interdependence between both ventricles. Failure of the left ventricle ( $L V)$ can lead to $R V$ failure. Impaired function of the $R V$ significantly worsens the prognosis in patients after myocardial infarction and with LV failure. Permanent atrial fibrillation $(A F)$ is one of the most common arrhythmia in patients with depressed RV function. Frequent coexistence of chronic heart failure $(C H F)$ and $A F$ causes overlapping of the arrhythmia and $R V$ dysfunction in the setting of CHF. They may lead to hemodynamic compromise and worsen prognosis in patients with chronic $R V$ failure of various etiologies. $R V$ structure and function can be assessed in $2 D, 3 D$ echocardiography, cardiac magnetic resonance imaging and computed tomography. (Cardiol J 2013; 20, 3: 220-226)
\end{abstract}

Key words: right ventricle, chronic heart failure, atrial fibrillation, CRT, echocardiography

\section{Introduction}

Dysfunctions of both ventricles often coexist in chronic heart failure (CHF) patients. Left ventricular (LV) failure can lead to right ventricular (RV) dysfunction. Occurrence of RV failure worsens prognosis in patients with dysfunctional LV. The aim of this paper is to reflect current reference on $\mathrm{RV}$ performance as a consequence of developing $\mathrm{CHF}$ and atrial fibrillation (AF). Existing dysfunction or exposure to RV overload can be a substrate for AF occurrence as well. Factors that determine RV function under normal conditions and as a consequence of developing $\mathrm{CHF}$ and/or $\mathrm{AF}$ are discussed below. Methods of right heart imaging are presented. The role of RV function at different stages of $\mathrm{CHF}$ seems to be underestimated and little attention has been paid so far to its function in $\mathrm{AF}$.

\section{Right ventricular function under normal conditions}

Function of the RV depends on its structure and myofibers architecture. The ventricle is triangular or crescent-shaped and is built of the inlet, the apical myocardium and the outlet tract. Its relatively thin myocardium is composed of a superficial circumferential layer, which continues to the $\mathrm{LV}$, and a deep longitudinal layer which facilitates shortening of the ventricle in its long axis during the systole. Other mechanisms that enable RV contraction are concentric inwards motion of the free wall and traction on the superficial fibers of the free wall secondary to the systole of the LV [1-3].

Due to elastance of the thin RV myocardium and low resistance of highly distensible pulmonary vessels, pressures in the right side of the heart are significantly lower and the RV is more dependent

Address for correspondence: Dr Ewa Majos, MD, $2^{\text {nd }}$ Ischemic Heart Disease Department, Institute of Cardiology, ul. Spartańska 1, 02-637 Warszawa, Poland, tel. + 482234340 50, fax: +48 2284495 10, e-mail: ewamjs@gmail.com Received: 28.10.2012 Accepted: 30.01.2013 
on the afterload than the LV. Preload determines function of both ventricles [3-5].

Beside the preload, afterload and muscle contractility, $\mathrm{RV}$ function is determined by the heart rhythm, RV filling time, RV systolic synchrony and interdependence between both ventricles. Maintenance of sinus rhythm and atrio-ventricular synchrony is crucial for RV function especially in chronic RV failure and acute RV infarction $[1,4]$. It was shown, that preserved function of both atria affects the RV more than the LV [2]. Ventricular interdependence is mainly dependent on function of the intraventricular septum. Studies in animal models proved that from $20 \%$ to $40 \%$ of systolic volume and pressure of the RV are generated by the LV systole. Diastolic interdependence is significantly marked under pathological conditions when volume or pressure overloaded RV shifts the intraventricular septum into the LV chamber and reduces the $L V$ preload and cardiac output. On the other hand, volume or pressure of overloaded LV causes pressure increase in the RV $[1,6]$.

\section{Right ventricular function in chronic heart failure}

Ventricular interdependence implies the fact that dysfunctions of both ventricles often coexist. LV failure can lead to development of RV failure. On the other hand, impaired function of the RV significantly worsens the prognosis in patients with $\mathrm{LV}$ failure after myocardial infarction (MI) [5, 7]. Substudy from the VALIANT ECHO study in a group of 600 patients after MI with LV dysfunction demonstrated that decreased RV function prognoses worse outcome. Patients with reduced RV fractional area change (RVFAC) were at higher risk of all-cause mortality, death from cardiovascular events and sudden death. Additionally, they were more prone to develop CHF and were at higher risk of stroke than control individuals. Each 5\% decrease in baseline RVFAC was associated with a 1.53 (95\% CI 1.24-1.88) increased risk of fatal and nonfatal cardiovascular outcomes [8]. Substudy from the SAVE study in subjects after MI with impaired LV function showed that RV dysfunction was an independent risk factor of total mortality, cardiovascular mortality and occurrence of CHF. Each 5\% reduction in the RVFAC resulted in $16 \%$ increased odds of cardiovascular mortality [9]. Other studies showed, that in patients with advanced CHF and LV ejection fraction (LVEF) $<40 \%$ prognosis strongly depends on RV function. $\mathrm{RV}$ function influenced the total outcome in these patients more significantly than LV function $[10,11]$.
Coexistence of $\mathrm{CHF}$ and $\mathrm{AF}$ is frequent. This is mainly due to common pathogenesis of both conditions and the fact that one enhances development and triggers symptoms of the other. Thus, in clinical practice both states should often be considered and treated together [12-14]. It is estimated that $\mathrm{AF}$ occurs in up to $50 \%$ of patients with $\mathrm{CHF}$ depending on NYHA class [14]. In patients with arrhythmia, LV diastolic function is often worse and may result in deterioration of RV function [15]. Therefore, frequent coexistence of RV dysfunction and $\mathrm{AF}$ in the setting of $\mathrm{CHF}$ is probable.

Cardiac resynchronization therapy (CRT) is a method of treatment in advanced CHF. It involves simultaneous stimulation of the RV and the LV. CRT is well established in patients with sinus rhythm. According to current guidelines it may be also used in patients with permanent $\mathrm{AF}(\mathrm{IIb} / \mathrm{C})[16]$. $\mathrm{Pa}$ tients responding to the CRT experience improvement of the LV function, subsequent clinical status and reduction in number of hospitalizations due to CHF $[17,18]$. Improvement of parameters used for evaluation of the LV function is observed: LVEF, end-diastolic volume, end-diastolic dimension, end-systolic volume and, end-systolic dimension [19]. CRT upgrades RV function which can be confirmed in the traditional echocardiographic examination by Tei-index and tricuspid annual systolic motion (TAPSE) evaluation [20-23]. The use of tissue Doppler imaging led to similar conclusions [24]. Recent studies seem to give a contrary opinion on how CRT influences markedly depressed RV function. It was proven, that significantly weakened $\mathrm{RV}$ function predisposes to a lack of improvement after CRT in terms of NYHA class, 6-minute walking test distance and LVEF [25-27]. On the other hand, it was shown that CRT may lead to improvement of the RV function regardless of its volume and size [27].

It is still not clear whether patients with AF may benefit from CRT to the same extent as the ones without arrhythmia. A systemic meta-analysis of 23 observational studies involved 7495 patients with CRT. It showed that AF was associated with lower rate of clinical response and increased risk of death in comparison to individuals without AF [28]. Contrary to that, some papers suggest that in patients with permanent AF treated with CRT a significant clinical improvement, comparable to the sinus rhythm group, can be achieved [29-31]. The effect of CRT has not been compared between patients with paroxysmal and persistent AF. One of few reports regarding this subject showed, that CRT leads to similar 
prognostic, symptomatic and echocardiographic benefits in patients with paroxysmal, permanent $\mathrm{AF}$ and sinus rhythm [32]. On the other hand, lower incidence of AF episodes in patients with CRT and paroxysmal AF can be achieved as a result of improved $\mathrm{LV}$ and left atrial function $[33,34]$. Specific evaluation of the CRT benefit in patients with paroxysmal and permanent AF in the context of the RV function is lacking.

\section{The right heart in patients with atrial fibrillation}

Relationship between the dimensions and function of the right atrium and occurrence of $\mathrm{AF}$ is properly documented [35, 36]. Enlargement and remodeling of both atria predispose to development of AF. Studies using stress echocardiography showed the prognostic values of the right atrium area to maintain sinus rhythm after AF ablation [35]. However, on the basis of existing evidence, area of the right atrium can be used to assess prognosis only in patients with non-valvular AF [36].

$\mathrm{RV}$ function in patients with AF is not well defined, although the importance of heart rhythm for the RV function is well known [4, 37]. Elongation of the cardiac cycle, thus lengthening the RV filling time results in diastolic volume increase and consequently in increase in RV ejection fraction (RVEF) [4]. Study in a small group of patients showed that in permanent AF the RV function presented as RVFAC is dependent on the RR interval preceding it and on the mean heart rate [37]. In another study in patients with paroxysmal and persistent AF RV end-systolic diameter was markedly larger than in individuals without the arrhythmia (20 $\pm 2 \mathrm{~mm}$ in AF group vs. $17 \pm 2 \mathrm{~mm}$ in control group). RVEF in patients with $\mathrm{AF}$ was deteriorated in comparison to healthy individuals: $69 \pm 8 \%, 60 \pm 11 \%$ and $74 \pm 3 \%$ in paroxysmal, permanent and control group, respectively. End-diastolic diameter did not differ between the groups [38]. The results of this study showed, that level of NT-proBNP, which is a marker of cardiac dysfunction, correlated with RVEF and was significantly higher in patients with permanent AF [39].

\section{Atrial fibrillation in the failing right heart}

Atrial tachyarrhythmias are most common in patients with depressed RV function. Their occurrence may be one of clinical manifestations of RV failure [2].

The results of the ACAP-HF Program in a group of 900 patients showed that occurence of the RV failure in the course of decompensated chronic LV failure increases the risk of AF development. Weakened RV function was one of the strongest predictors of AF occurrence. Two thirds of AF cases occurred in patients with RV dysfunction. Moreover, patients with RV dysfunction had higher risk of cardiac readmission and mortality than control individuals: $4.7 \%$ year vs. $2.9 \% / y e a r$. Finally, patients with deteriorated RV function who developed $\mathrm{AF}$ proved to be the ones with the worst prognosis in the whole group [40]. In patients with RV infarction hemodynamic stabilization depends also on maintenance of sinus rhythm [41].

Atrial flutter or AF may also lead to hemodynamic compromise and worsen prognosis in patients with chronic RV failure, not only of ischemic etiology [2, 42-44]. In pulmonary hypertension the RV is exposed to pressure overload. Hemodynamic and clinical stabilization depends mainly on its function. In patients with chronic pulmonary hypertension persistent AF is associated with significantly increased risk of death due to RV failure. Occurrence of supraventricular arrhythmias is often preceded by deterioration of RV function. In one of the studies mortality due to $\mathrm{RV}$ failure in patients with permanent AF was approximately $80 \%$. By contrast, in patients in whom sinus rhythm was restored after first episode of AF, total mortality was 6\% [42].

Observation of patients with congenital heart defects showed that abnormalities of RV structure predispose to developing atrial arrhythmias more than left-sided lesions. In one of the studies, risk of development of any atrial arrhythmia was circa $61 \%$ in the RV-defect group and $55.4 \%$ in the LV-defect group [43]. An isolated atrial septal defect, if not corrected, may cause a long lasting volume overload of the RV. The RV tolerates volume overload better than pressure overload. Dilatation of its cavity tends to normalize within 1-24 months after closure of the defect. However, in some patients it may last for over 5 years and AF may develop even decades after successful closing procedure [44]. A large analysis in a group of over 38000 patients with various congenital heart defects affecting the right heart showed $15 \%$ prevalence of atrial arrhythmias in adult patients. Other studies reported from $25 \%$ to $30 \%$ arrhythmia prevalence. Risk factors for AF or other atrial arrhythmia development included age, $\mathrm{CHF}$ and right atrial size. The results of the studies showed that atrial arrhythmias are associated with 2.5 fold increased risk of morbidity and $50 \%$ increased risk of mortality $[45,46]$. 


\section{Right ventricular pacing and heart failure}

RV pacing creates specific clinical conditions that affect RV function and may induce $\mathrm{AF}$ and $\mathrm{CHF}$ $[47,48]$. As a non-physiological mode of pacing it causes a loss of atrio-ventricular systolic synchrony. It has been proven that RV pacing exacerbates $\mathrm{HF}$ in patients with LVEF < 40\% [49]. In post-MI patients, RV apical pacing was associated with a worsening of $\mathrm{LV}$ function, suggesting at the same time that among MI survivors, the need for pacemaker may be a marker of worse outcome [50]. Cumulative RV pacing $>2 \%$ and $\mathrm{EF}<40 \%$ are independent predictors of ventricular tachycardia/ /ventricular fibrillation occurrence, higher mortality rate and $\mathrm{HF}$ hospitalizations in patients after cardioverter-defibrillator implantation [51].

The deleterious effects of RV apical pacing have been attributed to the abnormal electrical and mechanical activation, secondary to this form of pacing. During RV apical pacing, the electrical wave front propagates mainly through the myocardium rather than via the His-Purkinje conduction system. This is characterized by wave front breakthrough at the interventricular septum and latest activation of the infero-posterior base of the LV. The paced region contracts early at a time of low load, but then it is stretched in systole as the lateral wall finally contracts. Asynchronous myocardial contraction significantly decreases the stroke volume and right-shifts the LV end-systolic pressure - volume relationship. Thus, RV apical pacing leads to ventricular dyssynchronization, systolic and diastolic ventricular dysfunction, increase of wall stress, and energetic inefficiency [52]. It also causes interventricular dyssynchronization, as the $\mathrm{RV}$ contracts earlier than in a physiological model.

It has been proven that right ventricular outlet tract (RVOT) pacing can reduce unfavorable effects and slow down cardiac remodeling caused by permanent RV pacing. In one of the studies clinical and echocardiographic benefits observed in the RVOT group after 7 years of pacing were reflected by lower NT-proBNP concentration in this group of patients [53].

$\mathrm{CHF}$, as a result of impaired LV function, has been given close attention, but $\mathrm{RV}$ function during $\mathrm{RV}$ pacing still requires further research.

\section{Imaging and assessment of right ventricular structure and function}

Cardiac magnetic resonance imaging (CMR) is a precise method of RV structure and function assessment. It enables accurate quantification of heart chambers volumes and calculation of myocardial mass from tracing epicardial and endocardial borders and multiplying them by myocardial density [54]. Valvular regurgitant volumes and shunt severity can also be precisely calculated using velocity-encoded cine images [55]. Intra-observer and inter-observer variability in CMR RV measurements ranges from $3 \%$ to $6 \%$ and $4 \%$ to $9 \%$, respectively [56, 57]. Because of its good spatial and temporal resolution, CMR-derived volumes and $\mathrm{EF}$ are considered the gold standard regarding other imaging modalities. However, CMR is not routinely used because of its still limited availability and high costs.

Computed tomography (CT) has also been considered a reliable method to assess the RV. One of the studies showed, that there was little variability between the measurements of RV by the two observers (kappa $=0.895-0.980, \mathrm{p}<0.05$ ). There was also good correlation between all parameters obtained by CT and CMR ( $p<0.001)$ : RV end-diastolic volume (RVEDV $108.5 \pm 21.9 \mathrm{~mL}$, $113.5 \pm 24.8 \mathrm{~mL}, \mathrm{r}=0.944)$, $\mathrm{RV}$ end-systolic volume (RVESV $69.8 \pm 33.4 \mathrm{~mL}, 73.2 \pm 35.4 \mathrm{~mL}, \mathrm{r}=$ $=0.972$ ), RV systolic volume (RVSV $39.0 \pm 13.2 \mathrm{~mL}$, $40.2 \pm 13.3 \mathrm{~mL}, \mathrm{r}=0.977)$, RV cardiac output $(\mathrm{RVCO} 2.6 \pm 0.71,2.6 \pm 0.71, \mathrm{r}=0.958)$, and there was no significant difference between $\mathrm{CT}$ and CMR measurements in RVEF $(38.8 \pm 19.1 \%, 39.1 \pm$ $\pm 19.3 \%, r=0.990, \mathrm{n}=50, \mathrm{t}=-0.677, \mathrm{p}>0.05)$. The results of the study suggest, that 320 -slice volume cardiac CT is an accurate non-invasive technique to evaluate RV function [58]. Other paper shows that cardiac CT fully quantifies LV size and function. However, RV quantification with cardiac $\mathrm{CT}$ requires optimized contrast opacification of the $\mathrm{RV}$ [59]. When compared to radionuclide ventriculography, CT seems to be a reliable method for assessment of the RV as well. It has been shown that RVEF can be accurately assessed with ECG-gated multidetector computed tomography (MDCT) using commercially available software [60].

RV can also be accurately evaluated by echocardiography. Simple and established method for determining global RV function in the traditional two-dimensional imaging is an evaluation of TAPSE measured in M-mode presentation of the apical 4-chamber view [61]. The method is reliable also in patients with $\mathrm{AF}$ [62]. Percentage change in the surface of the RV (RVFAC) can also be assessed [8]. It is possible to calculate the ratio of RV global function (MPI, myocardial performance index). Additionally, tissue Doppler imaging enables measurement of peak systolic velocity of the tricuspid 


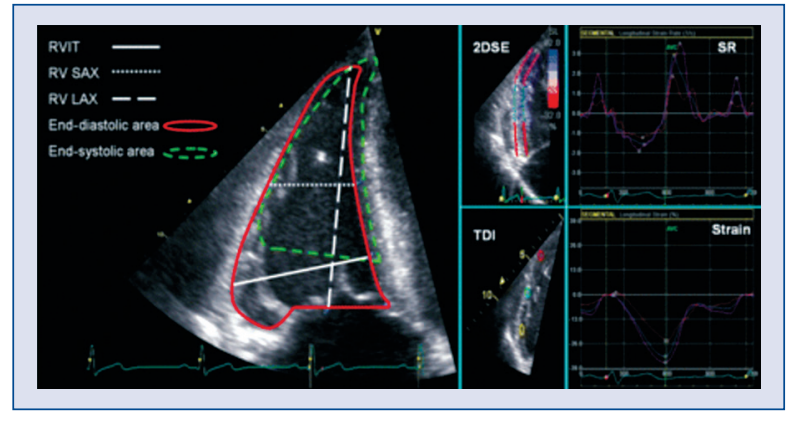

Figure 1. Right ventricular measurements [68].

annulus (S'), which is another complementary method for evaluating RV function [63]. RV free wall segmental motion and thickening are the points of evaluation as well. RV function can be assessed by eye-balling (Fig. 1).

A long-lasting overload of the RV can be indirectly estimated from the main pulmonary artery extension, measured in the parasternal short-vascular projection. The current flow through the pulmonary valve measured with use of pulse Doppler wave enables analysis of its spectrum and assessment of its acceleration time. Acceleration time is shortened when pulmonary arterial pressure is increased, RV systolic function is weakened and/or in paradoxical septal contraction occurring in acute conditions: acute pulmonary embolism and RV MI.

For the quantification of interventricular dyssynchrony, which is a predictor of outcome in CRT, conventional Doppler techniques are used. The electromechanical delay is calculated as the time from the onset of QRS complex to the onset of pulmonary systolic flow (RV electromechanical delay) or aortic systolic flow (LV electromechanical delay). The time difference between RV and LV electromechanical delay represents interventricular dyssynchrony [50].

Three-dimensional imaging echocardiography is a novel method which allows to obtain more reliable data on the structure and function of the RV, particularly on its RVESV and RVEDV. Additionally, the RVEF determined by this method shows good correlation with measurements in MRI [64-66].

According to current guidelines of the European Society of Echocardiography, assessment of the right heart structure should include RV, right atrium dimensions and areas in short, long parasternal axis and in apical 4-chamber view. RV
Table 1. Reference limits for recommended measures of right heart structure and function (from Guidelines for Echocardiographic Assessment of the right Heart in Adults: A Report from the American Society of Echocardiography) [67].

\begin{tabular}{|c|c|}
\hline Abnormal & $\begin{array}{l}\text { Chamber } \\
\text { dimensions }\end{array}$ \\
\hline RV basal diameter & $>4.2 \mathrm{~cm}$ \\
\hline RV subcostal wall thickness & $>0.5 \mathrm{~cm}$ \\
\hline RVOT PSAX distal diameter & $>2.7 \mathrm{~cm}$ \\
\hline RVOT PLAX proximal diameter & $>3.3 \mathrm{~cm}$ \\
\hline RA major dimension & $>5.3 \mathrm{~cm}$ \\
\hline RA minor dimension & $>4.4 \mathrm{~cm}$ \\
\hline RA end-systolic area & $>18 \mathrm{~cm}^{2}$ \\
\hline \multicolumn{2}{|l|}{ Systolic function } \\
\hline TAPSE & $<1.6 \mathrm{~cm}$ \\
\hline $\begin{array}{l}\text { Pulsed Doppler velocity peak at } \\
\text { the annulus }\end{array}$ & $<10 \mathrm{~cm} / \mathrm{s}$ \\
\hline Pulsed Doppler MPI & $>0.40$ \\
\hline Tissue Doppler MPI & $>0.55$ \\
\hline Fractional area change & $<35 \%$ \\
\hline \multicolumn{2}{|l|}{ Diastolic function } \\
\hline E/A ratio & $<0.8$ or $>2.1$ \\
\hline $\mathrm{E} / \mathrm{E}^{\prime}$ ratio & $>6$ \\
\hline Deceleration time & $<120 \mathrm{~ms}$ \\
\hline \multicolumn{2}{|l|}{ 3-dimensional imaging } \\
\hline RVEF & $<44 \%$ \\
\hline
\end{tabular}

RV - right ventricle; RVOT - right ventricular outlet tract; PLAX - parasternal long axis; PSAX — parasternal short axis; RA - right atrium; TAPSE - tricuspid annulus plane systolic excursion; MPI — myocardial performance index; RVEF — right ventricular ejection fraction

function should be evaluated with use of the above mentioned markers of its systolic and diastolic function and estimation of $\mathrm{EF}$ in the traditional $2 \mathrm{D}$ imaging. In addition, $\mathrm{EF}$ in $3 \mathrm{D}$ imaging can be assessed [67]. Table 1 presents RV dimensions and parameters of its function under normal conditions according to the guidelines.

\section{Conclusions}

$\mathrm{RV}$ function is crucial for outcome in patients with CHF, especially in patients with significant LV dysfunction. Its dysfunction often coexists with AF, not only in HF, but also in a number of conditions that depress RV acutely or chronically. $\mathrm{RV}$ structure can be precisely assessed with novel modalities of echocardiography, CT and magnetic resonance imaging.

Conflict of interest: none declared 


\section{References}

1. Haddad F, Hunt SA, Rosenhal DN, Murphy DJ. Right ventricular function in cardiovascular disease, part I: Anatomy, physiology, aging, and functional assessment of the right ventricle. Circulation, 2008; 117: 1436-1448.

2. Haddad F, Doyle R, Murphy DJ, Hunt SA. Right ventricular function in cardiovascular disease, part II: Pathophysiology, clinical importance, and management of right ventricular failure. Circulation, 2008; 117: 1717-1731.

3. Walker LA, Buttrick PM. The right ventricle: biologic insights and response to disease. Curr Cardiol Rev, 2009; 5: 22-28.

4. Dell'Italia LJ. Mechanism of postextrasystolic potentiation in the right ventricle. Am J Cardiol, 1990; 65: 736-741.

5. Santamore WP, Dell'Italia LJ. Ventricular interdependence: Significant left ventricular contributions to right ventricular systolic function. Prog Cardiovasc Dis, 1998; 40: 289-308.

6. Efthimiadis GK, Parharidis GE, Gemitzis KD et al. Doppler echocardiographic evaluation of right ventricular diastolic function in isolated valvular aortic stenosis. J Heart Valve Dis, 1999; 8: 261-269.

7. Mahmud M, Champion HC. Right ventricular failure complicating heart failure: Pathophysiology, significance, and management strategies. Curr Cardiol Rep, 2007; 9: 200-208.

8. Anavekar NS, Skali H, Bourgoun M et al. Usefulness of right ventricular fractional area change to predict death, heart failure, and stroke following myocardial infarction, from the VALIANT ECHO Study. Am J Cardiol, 2008; 101: 607-612.

9. Zornoff LA, Skali H, Pfeffer MA et al.; SAVE Investigators. Right ventricular dysfunction and risk of heart failure and mortality after myocardial infarction. J Am Coll Cardiol, 2002; 39: 1450-1455.

10. Meluzin J, Spinarova L, Hude P et al. Prognostic importance of various echocardiographic right ventricular functional parameters in patients with symptomatic heart failure. J Am Soc Echocardiogr, 2005; 18: 435-444.

11. Sun JP, James KB, Yang XS et al. Comparison of mortality rates and progression of left ventricular dysfunction in patients with idiopathic dilated cardiomyopathy and dilated versus nondilated right ventricular cavities. Am J Cardiol, 1997; 80: 1583-1587.

12. Deedwania PC, Lardizabal JA.Atrial fibrillation in heart failure: A comprehensive review. Am J Med, 2010; 123: 198-204.

13. Seiler J, Stevenson WG. Atrial fibrillation in congestive heart failure. Cardiol Rev, 2010; 18: 38-50.

14. Akoum N, Hamdan MH. Atrial fibrillation and congestive heart failure: A two-way street. Curr Heart Fail Rep, 2007; 4: 78-83.

15. Fung JW, Sanderson JE, Yip GW, Zhang Q, Yu CM. Impact of atrial fibrillation in heart failure with normal ejection fraction: A clinical and echocardiographic study. J Card Fail, 2007; 13: 649-655.

16. McMurray JJV, Aamopoulos S, Anker SD et al. ESC Guidelines for the diagnosis and treatment of acute and chronic heart failure 2012. The Task Force for the Diagnosis and Treatment of Acute and Chronic Heart Failure 2012 of the European Society of Cardiology. Developed in collaboration with the Heart Failure Association (HFA) of the ESC. Eur Heart J, 2012; 33: 1787-1847.

17. Vater M, Senges J Cardiac resynchronization therapy in patients with chronic heart failure: Pathophysiology and current experience. Am J Cardiovasc Drugs, 2002; 2: 219-226.

18. Turley AJ, Raja SG, Salhiyyah K, Nagarajan K. Does cardiac resynchronisation therapy improve survival and quality of life in patients with end-stage heart failure? Interact Cardiovasc Thorac Surg, 2008; 7: 1141-1146.

19. Bonanno C, Ometto R, Pasinato S, Finocchi G, La Vecchia L, Fontanelli A. Effects of cardiac resynchronization therapy on disease progression in patients with congestive heart failure. Ital Heart J, 2004; 5: 364-370.
20. Dubin AM, Feinstein JA, Reddy VM, Hanley FL, Van Hare GF, Rosenthal DN. Electrical resynchronization: A novel therapy for the failing right ventricle. Circulation, 2003; 18: 2287-2289.

21. Janousek J, Tomek V, Chaloupecky VA et al. Cardiac resynchronization therapy: A novel adjunct to the treatment and prevention of systemic right ventricular failure. J Am Coll Cardiol, 2004; 9: 1927-1931.

22. Miyazaki C, Yuasa T, Oh JK, Espinosa RE, Bruce CJ. Effects of cardiac resynchronization therapy on the Doppler Tei index. J Am Soc Echocardiogr, 2009; 22: 253-260.

23. Rajagopalan N, Suffoletto MS, Tanabe M et al. Right ventricular function following cardiac resynchronization therapy. Am J Cardiol, 2007; 100: 1434-1436.

24. Mabo P, Vignat N, De Place $C$ et al. Acute effects of biventricular pacing on right ventricular function assessed by tissue Doppler imaging. Europace, 2007; 9: 108-112.

25. Alpendurada F, Guha K, Sharma R et al. Right ventricular dysfunction is a predictor of non-response and clinical outcome following cardiac resynchronization therapy. J Cardiovasc Magn Reson, 2011; 13: 68.

26. Scuteri L, Rordorf R, Marsan NA et al. Relevance of echocardiographic evaluation of right ventricular function in patients undergoing cardiac resynchronization therapy. Pacing Clin Electrophysiol, 2009; 32: 1040-1049.

27. Ogunyankin KO, Puthumana JJ. Effect of cardiac resynchronization therapy on right ventricular function. Curr Opin Cardiol, 2010; 25: 464-468.

28. Wilton SB, Leung AA, Ghali WA, Farsi P, Exner DV. Outcomes of cardiac resynchronization therapy in patients with versus those without atrial fibrillation: a systematic review and meta-analysis. Heart Rhythm, 2011; 8: 1088-1094.

29. Schutte F, Luedorff G, Grove R, Kranig W, Thale J. Atrioventricular node ablation is not a prerequisite for cardiac resynchronization therapy in patients with chronic atrial fibrillation. Cardiol J, 2009; 16: 246-249.

30. Tolosana JM, Arnau AM, Madrid AH et al.; SPARE II Investigators (Spanish Atrial Resynchronisation Study II). Cardiac resynchronization therapy in patients with permanent atrial fibrillation. Is it mandatory to ablate the atrioventricular junction to obtain a good response? Eur Heart Fail, 2012; 14: 635-641.

31. Luedorff G, Grove R, Kowalski M, Wolff E, Thale J, Kranig W. Impact of chronic atrial fibrillation in patients with severe heart failure and indication for CRT: Data of two registries with 711 patients (1999-2006 and 2007-6/2008). Herzschrittmacherther Elektrophysiol, 2011; 22: 226-232.

32. Khadjooi K, Foley PW, Chalil S et al. Long-term effects of cardiac resynchronisation therapy in patients with atrial fibrillation. Heart, 2008; 94: 879-883.

33. Ding LG, Hua W, Chu JM et al. Improvement of P-wave dispersion is associated with a lower incidence of atrial fibrillation after cardiac resynchronization therapy. Chin Med (Engl), 2012; 125: 990-994.

34. Fung JW, Yip GW, Zhang Q et al. Improvement of left atrial function is associated with lower incidence of atrial fibrillation and mortality after cardiac resynchronization therapy. Heart Rhythm, 2008; 5: 780-786.

35. Houltz B, Johansson B, Berglin E, Karlsson T, Edvardsson N, Wandt B. Left ventricular diastolic function and right atrial size are important rhythm outcome predictors after intraoperative ablation for atrial fibrillation. Echocardiography, 2010; 27: 961-968.

36. Bilge M, Eryonucu B, Güler N, Erkoç R. Right atrial appendage function in patients with chronic nonvalvular atrial fibrillation. Jpn Heart J, 2000; 41: 451-462. 
37. Chou SH, Kuo CT, Hsu LA, Ho WJ, Wang CL. Single-beat determination of right ventricular function in patients with atrial fibrillation. Echocardiography, 2010; 27: 1188-1193.

38. Celebi O, Agactigen A, Sahin T et al. Right ventricular functions in patients with permanent and paroxismal atrial fibrillation. Eur J Echocardiogr, 2005; 6 (suppl. 1): S24.

39. Celebi O, Agactigen A, Sahin T et al. Brain natiuretic peptide levels and right ventricular function in paroxysmal and permanent atrial fibrillation. Eur J Echocardiogr, 2005; 6 (suppl. 1): S24.

40. Aziz EF, Kukin M, Javed F et al. Right ventricular dysfunction is a strong predictor of developing atrial fibrillation in acutely decompensated heart failure patients, ACAP-HF data analysis. J Card Fail, 2010; 16: 827-834.

41. O'Rourke RA, Dell'Italia LJ. Diagnosis and management of right ventricular myocardial infarction. Curr Probl Cardiol, 2004; 29: 6-47.

42. Tongers J, Schwerdtfeger B, Klein G et al. Incidence and clinical relevance of supraventricular tachyarrhythmias in pulmonary hypertension. Am Heart J, 2007; 153: 127-132.

43. Bernier M, Marelli AJ, Pilote L et al. Atrial arrhythmias in adult patients with right- versus left-sided congenital heart disease anomalies. Am J Cardiol, 2010; 106: 547-551.

44. Davlouros PA, Niwa K, Webb G, Gatzoulis MA. The right ventricle in congenital heart disease. Heart, 2006; 92 (suppl. 1): i27-i38.

45. Darby AE, Dimarco JP. Management of atrial fibrillation in patients with structural heart disease. Circulation, 2012; 125: 945-957.

46. Bouchardy J, Therrien J, Pilote L et al. AJ. Atrial arrhythmias in adults with congenital heart disease. Circulation, 2009; 120: 1679-1686.

47. Zhang XH, Chen $\mathrm{H}$, Siu $\mathrm{CW}$ et al. HF New-onset heart failure after permanent right ventricular apical pacing in patients with acquired high-grade atrioventricular block and normal left ventricular function. J Cardiovasc Electrophysiol, 2008; 19: 136-141.

48. Varma N, Jia P, Ramanathan C, Rudy Y. RV electrical activation in heart failure during right, left, and biventricular pacing. J Am Coll Cardiol Cardiovasc Imaging, 2010; 6: 567-575.

49. Ritter O, Koller ML, Fey B et al. Progression of heart failure in right univentricular pacing compared to biventricular pacing. Int J Cardiol, 2006; 110: 359-365.

50. Muto C, Ascione L, Canciello M et al. Effect of right ventricular apical pacing in survivors of myocardial infarction. Pacing Clin Electrophysiol, 2009; 32 (suppl. 1): S173-S176.

51. Gardiwal A, Yu H, Oswald $\mathrm{H}$ et al. Right ventricular pacing is an independent predictor for ventricular tachycardia/ventricular fibrillation occurrence and heart failure events in patients with an implantable cardioverter-defibrillator. Europace, 2008; 10: 358-363.

52. Brenyo A, Goldberg I, Barsheshet A. The downside of right ventricular apical pacing. Indian Pacing Electrophysiol J, 2012; 12: 102-113.

53. Lewicka-Nowak E, Dabrowska-Kugacka A, Tybura S et al. Right ventricular apex versus right ventricular outflow tract pacing: Prospective, randomised, long-term clinical and echocardiographic evaluation. Kardiol Pol, 2006; 64: 1082-1091.

54. Greyson CR. Evaluation of right ventricular function. Curr Cardiol Rep, 2011; 13: 194-202.

55. Goetschalckx K, Rademakers F, Bogaert J. Right ventricular function by MRI. Curr Opin Cardiol, 2010; 25: 451-455.
56. Luijnenburg SE, Robbers-Visser D, Moelker A, Vliegen HW, Mulder BJ, Helbing WA. Intra-observer and interobserver variability of biventricular function, volumes and mass in patients with congenital heart disease measured by CMR imaging. Int J Cardiovasc Imaging, 2010; 26: 57-64.

57. Maceira AM, Prasad SK, Khan M, Pennell DJ. Reference right ventricular systolic and diastolic function normalized to age, gender and body surface area from steady-state free precession cardiovascular magnetic resonance. Eur Heart J, 2006; 27: 2879-2888.

58. Huang X, Pu X, Dou R et al. Assessment of right ventricular function with 320-slice volume cardiac CT: comparison with cardiac magnetic resonance imaging. Int J Cardiovasc Imaging, 2012; 28 (suppl. 2): 87-92.

59. Raman SV, Shah M, McCarthy B, Ferketich AL. Multi-detector row cardiac computed tomography accurately quantifies right and left ventricular size and function compared with cardiac magnetic resonance. Am Heart J, 2006; 151: 736-744.

60. Remy-Jardin M, Delhaye D, Teisseire A, Hossein-Foucher C, Duhamel A, Remy J. MDCT of right ventricular function: Impact of methodologic approach in estimation of right ventricular ejection fraction, part 2. Am J Roentgenol, 2006; 187: 1605-1609.

61. Miller D, Farah MG, Liner A, Fox K, Schluchter M, Hoit BD. The relation between quantitative right ventricular ejection fraction and indices of tricuspid annular motion and myocardial performance. J Am Soc Echocardiogr, 2004; 17: 443-447.

62. Kim H, Jung C, Yoon HJ, Park HS et al. Prognostic Value of Tricuspid Annular Tissue Doppler Velocity in Heart Failure with Atrial Fibrillation. J Am Soc Echocardiogr, 2012; 25: 436-443.

63. Belenkov IuN, Agmanova ET. Role of tissue Doppler echocardiography in the diagnosis of right ventricular dysfunction in patients with chronic functional class I-IV heart failure. Kardiologia, 2007; 47: 18-21.

64. van der Zwaan HB, Helbing WA, Boersma E et al. Usefulness of real-time three-dimensional echocardiography to identify right ventricular dysfunction in patients with congenital heart disease. Am J Cardiol, 2010; 106: 843-850.

65. Grewal J, Majdalany D, Syed I, Pellikka P, Warnes CA. Three-dimensional echocardiographic assessment of right ventricular volume and function in adult patients with congenital heart disease: Comparison with magnetic resonance imaging. J Am Soc Echocardiogr, 2010; 23: 127-133.

66. Nesser HJ, Tkalec W, Patel AR et al. Quantitation of right ventricular volumes and ejection fraction by three-dimensional echocardiography in patients: comparison with magnetic resonance imaging and radionuclide ventriculography. Echocardiography, 2006; 23: 666-680.

67. Rudski LG, Lai WW, Afilalo J et al. Guidelines for Echocardiographic Assessment of the right Heart in Adults: A Report from the American Society of Echocardiography endorsed by the American Society of Echocardiography a registered branch of the American Society of Cardiology and the Canadian Society of Echocardiography. J Am Soc Echocardiogr, 2010; 23: 685-713.

68. Fang F, Zhang Q, Chan JYS et al. Deleterious effect of right ventricular apical pacing on left ventricular diastolic function and the impact of pre-existing diastolic disease. Eur Heart J, doi:10.1093/ eurheartj/ehr118. 